\title{
Integrated Elastomer Fluidic Lab-on-a-chip - Surface Patterning and DNA Diagnostics
}

\author{
Hou-Pu Chou", Marc A. Unger ${ }^{+}$, Axel Scherer ${ }^{*+}$, and Stephen R. Quake ${ }^{+}$ \\ Departments of Electrical Engineering ${ }^{*}$ and Applied Physics ${ }^{+}$, Caltech, \\ Pasadena, CA 91125, USA
}

\begin{abstract}
We recently developed a method of multilayer fabrication for elastomeric devices, which we used to fabricate monolithic active valves and pumps. Here we describe efforts to use these pumps and valves in an integrated DNA diagnostic chip and show results of a key component, surface patterning, with two different kind of surface chemistries by using similar elastomeric channel devices. Flow control, reagent metering, in-line mixing and loop circulations are also demonstrated.
\end{abstract}

\section{INTRODUCTION}

Several techniques are currently being developed towards the goal of an integrated fluidic lab-on-a-chip ${ }^{[1-6]}$. Among these, monolithic microvalves and micropumps made from silicone elastomer $^{[5]}$ have great potential because of their simplicity, robustness, easy fabrication and low cost. Here, we describe some new results and extensions to our previous work as part of our ultimate goal of creating an integrated DN $\Lambda$ diagnostic chip.

We are interested in using microfabricated chips to measure gene expression and detect the presence of pathogenic DNA. DNA expression arrays have proved to be a useful tool in studying gene expression in a variety of organisms, including yeast, worm, mouse and human ${ }^{[7]}$. The sensitivity of such arrays is limited in part by the diffusion of target DNA to the probes that are anchored on the surface ${ }^{[8]}$. A better approach is to use microfluidic devices in order to pump solutions of target DNA over a set of anchored probes in order to ensure that all of the target DNA is exposed to each of the probes. This would provide increased sensitivity as well as decreasing the amount of time needed for hybridization. Chips with high sensitivity would also be useful for measuring single cell gene expression. This higher sensitivity may eliminate the need for PCR in many cases of pathogen detection and therefore make it possible to do multiple disease diagnosis with one integrated lab-on-a-chip.

Making such chips requires a number of important technological advances in the current state of the art of microfluidics. First, one needs to be ablc to fabricatc microfluidic devices in a way that is compatible with the delicate surface chemistry required to anchor or synthesize DNA probes on a chip. Second, one must be able to effect the desired patterning or surface chemistry. Third, one must be able to manipulate small amounts of material and perform the necessary biochemical reactions on chip. Finally, one needs to be able to pump the targets over the probes.

The first two issues we have addressed by using "soft lithography", which in part refers to the notion of fabricating channel systems out of soft elastomeric materials using replication molding techniques ${ }^{[9]}$. We have found that elastomeric devices provide a number of important advantages over conventional micromachining, such as ease of fabrication, room temperature sealing of devices to glass substrates, good optical properties, and low materials cost, especially compared to single crystal silicon: $\sim \$ .05 / \mathrm{cm}^{3}$ vs. $\sim \$ 2.50 / \mathrm{cm}^{3}$. Microfluidic networks fabricated in such a manner can easily be sealed to substrates with delicate surface chemistry. Another aspect of soft lithography we have taken advantage of is the ability to chemically pattern surfaces using fluid flow ${ }^{[10,11]}$. The third and final issues we have addressed by developing a multilayer fabrication process in silicone elastomer. This multilayer process allows easy fabrication of devices with moving parts, including microfluidic valves and pumps $^{[5]}$.

Here we describe results in which we chemically pattern surfaces with biotin/avidin and DNA using fluidic networks in a way that is compatible with further fluidic processing. We also show how pumps can be incorporated into an integrated device to both meter reagents and pump fluid in a closed loop. Rotary flow in a closed loop cannot be achieved by electrophoresis or electroosmotic flow used in most common lab-on-a-chips because of the existence of two electric polarities.

\section{DEVICE DESIGN AND FABRICATION}

Multilayer soft lithography is a process recently developed in our lab in order to make 3-D monolithic elastomer devices with a combination of air and fluid channels ${ }^{[5]}$. When an air channel passes above another fluid channel, the thin membrane between these two channels becomes a valve. By applying air pressure in the air channel, the membrane collapses and stops the fluid flow. Releasing the pressure then re-opens this valve. Three valves in series become a peristaltic pump when an appropriate on/off air pressures are applied in a sequence. For example, 100, 110, 010, $011,001,101$ pumps water to the right, where 1 and 0 represent closed or open valves respectively. A schematic diagram is shown in Figure 1. A brief description of the fabrication process follows.

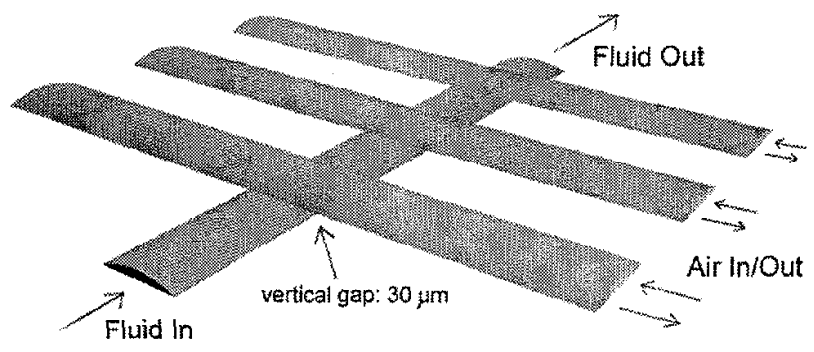

Figure 1. Schematic diagram of a peristaltic pump with three valves on top of a fluid channel.

Air and fluid mother molds were fabricated on silicon wafers by photolithography. Photoresist (Shipley SJR5740) was spun onto the silicon substrate at spin rates corresponding to the desired channel heights. After photolithography, intrusive channels made of photoresist were formed. Fluid channel molds were baked on a hot plate of $200^{\circ} \mathrm{C}$ for 30 minutes so that the photoresist could reflow and form a rounded shape, which is important for complete valve closure ${ }^{[5]}$. A one minute trimethylchlorosilane (TMCS) 


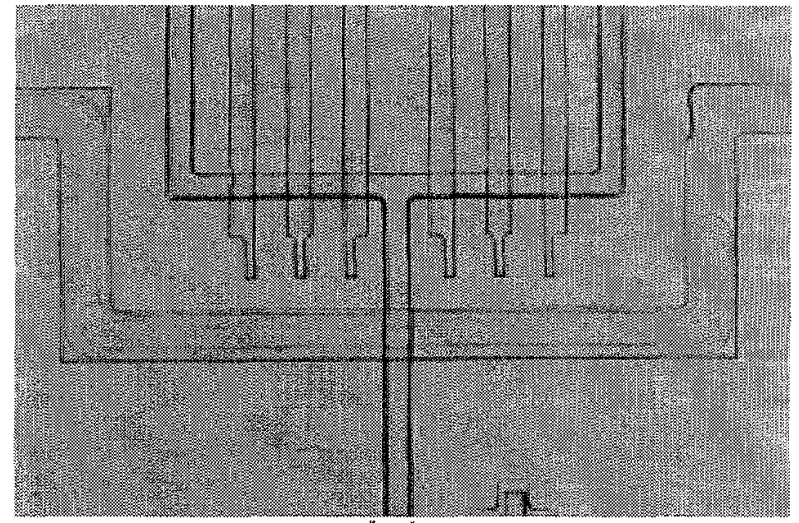

(a).

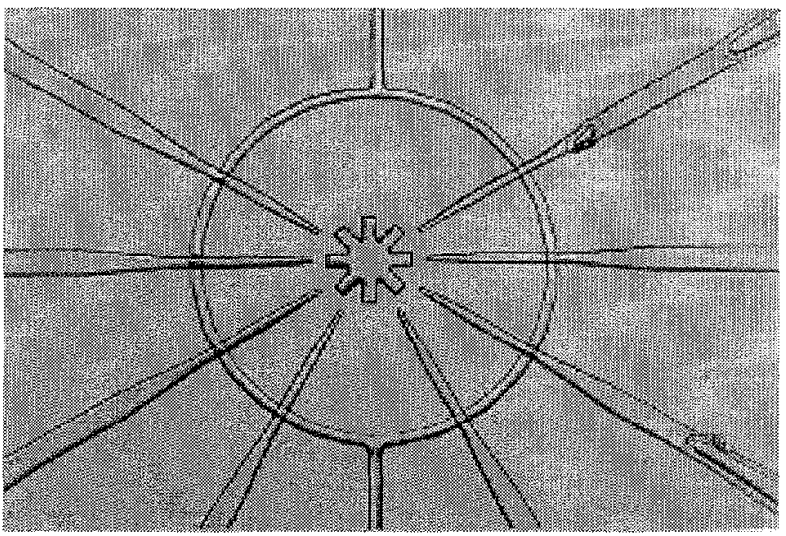

(b).

Figure 2. Snapshots of a two-layer DNA diagnosis chip. (a) shows the input mixing T-shaped fluid channel and six corresponding control microvalves. The wide 100- $\mu \mathrm{m}$ air channel beneath is used to close the inlet when the peristaltic pump at the loop starts operating for hybridization. (b) shows the center fluid loop for DNA hybridization. Any three of the finger air channels form a peristaltic pump. Fluid channel width: $50 \mu \mathrm{m}$.

vapor treatment was applied to these molds before each RTV replication process to prevent adhesion of cured RTV to the photoresist. With this protective coating, molds can be reused many times.

30:1 GE-RTV 615A:615B was spun on a fluid channel mold at 2,000 RPM, which covers the photoresist channel and leave a thin membrane on top of it. At the same time, 3:1 GE-RTV 615A:615B was poured onto an air channel mold. After baking both in an oven of $80^{\circ} \mathrm{C}$ for 20 minutes, the block of 3:1 RTV with air channels at the bottom was peeled off from the second mold. Air supply through-holes were punched. Aligned to the fluid pattern under a microscope, it was then pressed against the thin 30:1 RTV on the first mold. A post-bake of an hour at $80^{\circ} \mathrm{C}$ made the two silicone pieces chemically bond to each other. After peeling it off from the mold and punching the fluid through-holes, the monolithic RTV device could seal hermetically to a glass cover slip. In principle this glass cover slip could be chemically patterned in advance, as we describe in the next section, to make an active diagnostic chip. If high-resolution transparency photomasks were used (minimum feature size: $\sim 10 \mu \mathrm{m}$ ), the whole process from the design to the final products would be accomplished in just a day.
Snapshots of a final assembled device are shown in Figure 2. The central loop is the key component, at which the DNA hybridization probes are laid down along the ring on top of the glass substrate. DNA samples and later fluorescent intercalating dyes can enter from the two branches of the top T-channel. On/off statcs of each microvalves are controlled by external pneumatic valves (Lee LHDA1211111H) which either apply 100-kPa air pressure to the microvalves or vent them to the atmosphere. A maximum cycling frequency of $75 \mathrm{~Hz}$ has been demonstrated with complete opening and closing of the valves ${ }^{[4]}$.

\section{SURFACE PATTERNING}

We developed two independent methods of surface patterning. The first method allows patterning of the protein streptavidin, a common biochemical "glue" that binds biotin with nearly covalent strength. With the streptavidin surfaces, one can selectively anchor biotin-labeled reagents, including proteins and nucleic acids. The second method allows direct attachment of amine-modified DNA molecules to the surface using a commercially available surface chemistry from the company Surmodics.

In the first method, we derivatized half of the surface of a cover slip (VWR \#1) with biotin. ${ }^{[12]}$ We then attached channels and flowed avidin-fluorescein conjugate down them, after which we flushed the channels with water, removed the channels, and llushed the entire cover slip. The avidin molecules bound to the derivatized part of the surface with high affinity in the regions defined by the channels, forming fluorescent stripes as shown on Figure 3 (a). The regions which were not derivatized with biotin function as a control and showed a much lower level of avidin binding.

We were also able to perform successive patterning steps to the surface. In this case we patterned the surface with nonfluorescent streptavidin by bonding an elastomeric device with channels to the substrate and flowing the streptavidin down the channels. As before, the streptavidin bound selectively to the surfaces that were exposed to the channels, and not to the surface covered by the elastomer. Since streptavidin is a tetramer, each molecule has at least two exposed groups free to bind more biotin. We demonstrated this by then removing the elastomeric channcls and re-bonding in an orientation that was rotated by 90 degrees. We flowed biotin-fluorescein conjugates down the channels and then washed with water. From the images in figure 3 (b), it is clear that the fluorescent biotin binds selectively to the regions that are derivatized with streptavidin.

We also prepared surfaces patterned with DNA using commercially available silanized slides (Surmodics 3D-Link). DNA samples were prepared by PCR of a $2 \mathrm{kpb}$ region of lambda phage DNA using amino-terminated primers. The DNA was attached in situ by flowing it through an elastomeric channel replica made from the air channel mold of the diagnosis chip, i.e. the finger pattern in Figure 2 (b). After overnight incubation, the elastomeric device was peeled off from the slide, and washing and immobilization steps were followed according to the 3D-Link protocol. To show that the DNA was attached to the surface, we aligned and attached a diagnostic RTV device (as shown in Figure 2) to the same slide. Then we flowed the DNA intercalating dye PicoGreen (Molecular Probes P-7581) through the bottom fluid channel, of which the central ring intersected with every DNA finger pattern on the slide. The intersection of the channels fluoresced, as shown in Figure 3 (c). 

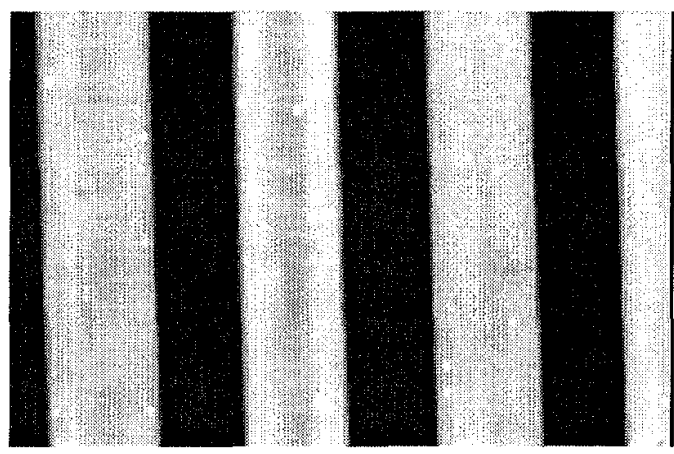

(a).

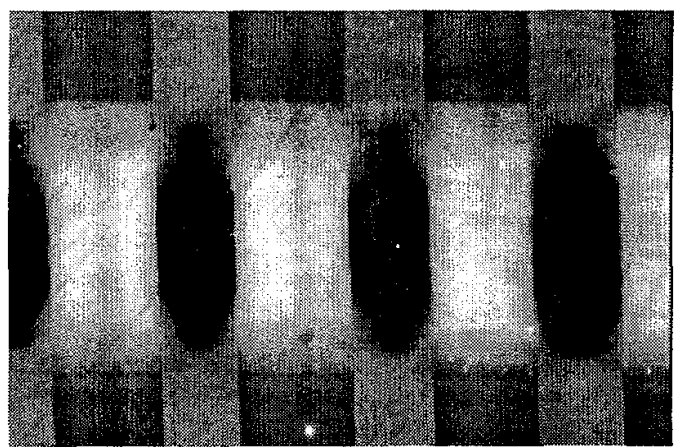

(b).

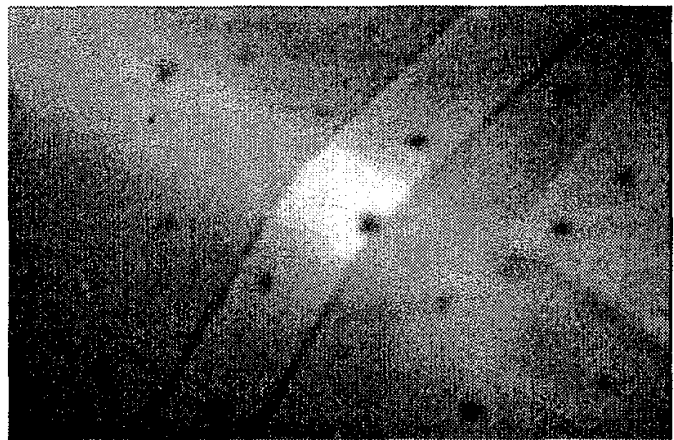

(c)

Figure 3. Images of chemically patterned cover slips. (a) A line pattern obtained by flowing avidin-fluorescein conjugates vertically on a biotinylated cover slip. (b) $A$ checkerboard pattern obtained by flowing streptavidin horizontally $(200 \mu \mathrm{m})$ and biotin-fluorescein conjugates vertically $(100 \mu \mathrm{m})$. (c) DNA patterned on a Surmodics slide lights up when fluorescent dye PicoGreen was flowing in the central ring loop. Top-left to bottom-right was DNA with slightly auto-fluorescence. Top-right to bottom-left was part of the central ring of a diagnosis chip.

\section{RESULTS}

We have demonstrated two methods of chemical patterning of surfaces using soft lithography, and shown that the chemical patterns can also be interfaced with elastomeric microfluidics. These methods are the starting point for proof of principle of DNA diagnostics, and provide the foundation to demonstrate sensitive and selective DNA detection on chip. It is a straightforward extension to use protein-binding assays with these fluidic systems as well. One issue that remains to be addressed is the strength of adhesion - the elastomer adheres to derivatized surfaces, but not with the same strength as to clean glass.

Our prototype DNA diagnostic chip has a junction for mixing and metering reagents, which then leads into a fluidic loop. The probe molecules will be anchored in the loop so that the targets can circulate around. The loop has peristaltic pumps around it to control circulation. The fluidic connections into and out of the loop are controlled by input and output valves, respectively.

We have demonstrated mixing and metering in the first part of the chip. A solution containing fluorescent dye can be mixed with an aqueous solution - the flows can be alternated with two different valve-firing schemes. If the valves arc opencd and closed in synchrony, the fluid mixing is controlled by diffusion. One observes two segregated flows when the valves are open, which quickly mix by diffusion when the valves are closed. If the valves are opened and closed alternately, then slugs of fluid are injected into the stream

We have also pumped fluid within the closed loop using the peristaltic pumps on the perimeter. The channels were loaded with fluorescent beads $(2.5 \mu \mathrm{m}$ in diameter). The beads could be visualized as the fluid circulated around the loop and clearly showed rotary motion with no net flux into or out of the loop. Thus reagents can be repetitively exposed to diagnostic probes anchored on the surface, and their binding will not be limited by diffusion. All target DNA in a sample should be eventually captured by their corresponding probes after several passages. Such a device can also be used to rapidly mix viscous liquids, since the parabolic flow profile of the fluid will tend to "wrap" the two fluids around each other.

\section{CONCLUSION}

Here we have demonstrated elements of an active DNA diagnosis chip using multilayer soft lithography. DNA fragments can be patterned on a glass substrate with appropriate surface chemistry combination and by using elastomeric fluidic channels. A monolithic elastomeric diagnosis device can then be aligned and attached to the derivatized surface. DNA samples can be brought in from the top, circulated several times in the hybridization loop and expelled from the bottom outlet. Fluorescent dye used to determine the hybridization can be brought in from another input channel. Fast mixing can also be done with the active pumping mechanism. Laminar flow and diffusion limitation in this lowReynolds number regime can be overcome easily by this active pumping agitation.

With the power of multilayer soft lithography, many complicated functions can easily be designed and fabricated as we have shown here. We anticipate that more active lab-on-a-chip devices will be developed rapidly. The problem of buffer depletion due to electrolysis in electro-osmotic flow control does not exist in these devices. Rotary mixing, rapid cell sorting, precise chemical metering and time-controlled reactions are all possible.

\section{REFERENCES}

1. A. T. Woolley, K. Q. Lao, A. N. Glazer and R. A. Mathics, "Capillary Electrophoresis Chips with Integrated Electrochemical Detection," Analytical Chemistry, 70 (4), pp. 684 (1998).

2. N. H. Chiem and D. J. Harrison, "Microchip Systems for Immunoassay: an Integrated Immunoreactor with Electrophoretic Separation for Serum Theophylline Determination," Clinical Chemistry, 44 (3), 591 (1998). 
3. M. U. Kopp, A. J. de Mello and A. Manz, "Chemical Amplification: Continuous-flow PCR on a Chip," Science, 280 (5366), 1046 (1998).

4. L. C. Waters, S. C. Jacobson, N. Kroutchinina, J. Khandurina, R. S. Foote, and J. M. Ramsey, "Microchip devices for cell lysis, multiplex PCR amplification, and electrophoretic sizing," Analytical Chemistry., 70 (1), 158 (1998).

5. M. A. Unger, H. P. Chou, T. Thorsen, A. Scherer and S. R. Quake, "Monolithic Microfabricated Valves and Pumps Using Multi-layer Soft Lithography," Science in press, (2000).

6. A. Y. Fu, C. Spence, A. Scherer, F. H. Arnold and S. R. Quake, "A Microfabricated Fluorescence-Activated Cell Sorter," Nature Biotechnology, 17, 1109 (1999).

7. G. Ramsay, "DNA chips: State-of-the art," Nature Biotechnology, 16, 40 (1998).

8. Diffusion length $l=\sqrt{D t}$, where $D$ is the diffusion constant and $t$ is the time. Let $D$ be $10^{-7} \mathrm{~cm}^{2} / \mathrm{s}$ for a typical $1 \mathrm{kbp} \mathrm{DNA}$ and $t$ be an hour. Then, the diffusion length $l$ would be 0.19 $\mathrm{mm}$. So, if only passive diffusion is used, each hybridization spot can only cover an area of about $0.4 \mathrm{~mm}$ in diameter. Even after a day, 24 hours, only target samples in an area of $\sim 2 \mathrm{~mm}$ in diameter can possibly reach a specific probe to give a positive signal. This is an extremely slow process for big molecules like DNA.

9. Y. N. Xia and G. M. Whitesides, "Soft Lithography", Angew. Chem. Int. Ed., 37, 550 (1998).

10. R. S. Kane, S. Takayama, E. Ostuni, D. E. Ingber, and G. M. Whiteside, "Patterning Proteins and Cells Using Soft Lithography," Biomaterials, 20 (23-24), 2363 (1999).

11. E. Delamarche, A. Bernard, H. Schmid, B. Michel, and H. Biebuyck, "Patterned Delivery of Immunoglobulins to Surfaces Using Microfluidic Networks," Science, 276, 779 (1997).

12. Marc A. Unger, Emil Kartalov, and Stephen R. Quake, manuscript in preparation. 